Research Paper

\title{
A single nucleotide polymorphism in CYPIBI leads to differential prostate cancer risk and telomere length
}

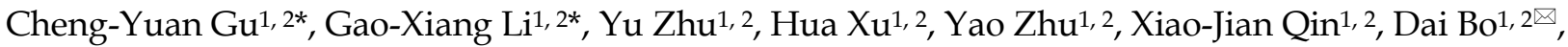 \\ Ding-Wei Ye $\mathrm{e}^{1,2 \bowtie}$ \\ 1. Department of Urology, Fudan University Shanghai Cancer Center, Shanghai, China; \\ 2. Department of Oncology, Shanghai Medical College, Fudan University, Shanghai, China. \\ * These authors contributed equally to this work. \\ $\triangle$ Corresponding authors: Bo Dai (bodai1978@126.com) and Ding-Wei Ye (dwyeli@163.com), Department of Urology, Fudan University Shanghai Cancer \\ Center, No. 270 Dong'an Road, Shanghai 200032, People's Republic of China. Tel: 86-21-64175590-2807; Fax: 86-21-64434556. \\ (c) Ivyspring International Publisher. This is an open access article distributed under the terms of the Creative Commons Attribution (CC BY-NC) license \\ (https://creativecommons.org/licenses/by-nc/4.0/). See http://ivyspring.com/terms for full terms and conditions.
}

Received: 2017.07.05; Accepted: 2017.10.09; Published: 2018.01.01

\begin{abstract}
BACKGROUND: Cytochrome P450 1B1 (CYPIB1) is a key enzyme in its oestrogen metabolism pathway, giving rise to hydroxylation and conjugation. Functionally relevant genetic variants within CYPIBI may affect the telomere length and subsequently lead to prostate carcinogenesis.

METHODS: We evaluated 8 CYPIBI tag single nucleotide polymorphisms (SNPs) in 1015 men with prostate cancer (PCa) and 1052 cancer-free controls, and calculated odds ratios (ORs) and $95 \%$ confidence intervals (Cls) to estimate their association with risk of PCa. The influence of CYPIBI SNPs on the relative telomere lengths was then appraised in peripheral blood leukocytes using real-time PCR.

RESULTS: CYPIBI rs 1056836 variant was associated with decreased risk of $\mathrm{PCa}$ [odds ratio (OR): 0.80 ; 95\% confidence interval $(\mathrm{Cl}): 0.68-0.99, P=0.041]$. Longer telomere length showed a significantly higher proportion of the CYPIBI rs 1056836 CG/GG genotypes, compared with that of the CC genotype (OR: $1.60,95 \% \mathrm{Cl}: 1.04-2.45)$.

CONCLUSION: Our findings suggest that genetic variants within CYPIBI may confer genetic susceptibility to $\mathrm{PCa}$ by altering telomere length.
\end{abstract}

Key words: CYP1B1; prostate cancer; single nucleotide polymorphism; susceptibility.

\section{Introduction}

Telomeres are specialised hexanucleotide repeats complexed to proteins. They are located at ends of linear chromosomes and protect their structural integrity ${ }^{[1]}$. Telomere length reduces with increasing chronological age and with accumulation of age-related comorbidities. Given that telomere shortening contributes to cellular senescence, telomere length represents a cellular marker of biological aging. A large number of studies suggest that telomere length is a marker of cancer risk and short leukocyte telomere length are believed to be causative events in malignant transformation ${ }^{[1]}$.

The hypothesis that estrogen influences telomere length came from several preclinical, observational and interventional studies[2].This phenomenon was explained by the finding that estrogen stimulates telomerase via estrogen response element present in the TERT promoter region. Additional protective effect of estrogen on telomere length is thought to be mediated through its reduction in oxidative stress ${ }^{[3]}$. Despite the well-known antioxidant role of estrogen, estrogen metabolites, on the other hand, may paradoxically lead to increased oxidative stress through the formation of depurinating DNA adducts and release of surplus amount of superoxide radicals ${ }^{[4]}$. Several single nucleotide polymorphisms (SNPs) within the estrogen metabolic pathway have been described to have functional effects on the corresponding enzymes, thereby compromising conjugation and redox buffering capacity of cells[5]. 
Candidates include CYP1B1, which catalyze the conversion of estrogens to reactive catechol estrogens. Furthermore, we described that a CYP1B1 SNP rs1056836 was significantly associated with biochemical recurrence after radical prostatectomy and relative CYP1B1 mRNA expression[6].

Encouraged by such findings we have now tested CYP1B1 SNPs for associations with risk of PCa in a case-control study. In addition, a real-time PCR based telomere assay was performed to investigate whether leukocyte telomere was in relation to CYP1B1 SNPs.

\section{Materials and Methods}

\section{Study Design and Population}

The study subjects were mostly from previously published case-control study[7]. Briefly, 1115 eligible patients recruited into this study were newly diagnosed and histopathologically confirmed primary prostate adenocarcinoma from Fudan University Shanghai Cancer Center (FUSCC) between January 2005 and January 2012, of whom 1015 (92\%) agreed to participate in this study. All cases had received no prior chemotherapy or radiotherapy upon recruitment. The tumor stage was determined according to criteria established by the American Joint Committee on Cancer (AJCC) tumor-node-metastasis (TNM) classification system [AJCC Staging Manual, sixth edition, 2002]. Histopathological grading of the specimens was performed according to the Gleason score system. The clinical information including Gleason score, serum PSA level at diagnosis and disease stage were abstracted from the archival medical records. In addition, 1143 age- $( \pm 5 y r)$ and geographical regions-matched cancer-free ethnic Han Chinese controls were recruited from the Taizhou longitudinal (TZL) study conducted during a similar time period. TZL study was a large prospective cohort initiated to explore the environmental and genetic risk factors for common non-communicable diseases. Individuals with a known test of serum PSA $>4$ $\mathrm{ng} / \mathrm{mL}$ present with or without abnormal digital rectal examination were excluded from the control group and those without response to the study participation were excluded $(n=91)$. All of the participants were interviewed with a questionnaire after a written informed consent was obtained. Blood samples were collected and processed as a routine practice by the FUSCC Tissue Bank (for cases) and the TZL study (for controls). This study was approved by the Institutional Review Board of FUSCC.

\section{SNP Selection and Genotyping}

Tagging SNPs were selected in the CYP1B1 gene using the Tagger algorithm with $r^{2} \geq 0.8$, and minor-allele frequencies $>0.05$ based on the HapMap population data for Han Chinese in Beijing. There were 9 tagging SNPs (rs1056836, rs10916, rs162562, rs2551188, rs9341250, rs9341266, rs9341248, rs162549, rs1056827).

All these nine SNPs were genotyped by the TaqMan real-time PCR method as described previously ${ }^{[8,9]}$. Briefly, DNA isolation was performed by using the Qiagen Blood DNA Mini KIT (Qiagen Inc., Valencia, CA) with the buffy-coat fraction of the blood samples donated by the participants. The results with $>99 \%$ call rates and $100 \%$ concordance for duplicated specimens were acceptable for further genotyping data analysis. SNP rs9341248 was removed because it did not conform to Hardy-Weinberg equilibrium (HWE) $(\mathrm{P}<0.05)$. Thus, a total of 8 SNPs were included for subsequent analysis.

\section{Measurement of Relative Telomere Length}

Relative telomere length measurements were available in 426 patients as described previously[9]. Briefly, telomere length as represented by the telomere repeat copy number to single copy gene (i.e., 36B4) copy number (T/S) ratio was measured using real-time quantitative PCR method on an Applied Biosystems 7900HT. The PCR reaction mixture consisted of SYBR Green Mastermix (Applied Biosystems), $100 \mathrm{nmol} / \mathrm{L}$ Tel-1, $900 \mathrm{nmol} / \mathrm{L}$ Tel-2, 400 $\mathrm{nmol} / \mathrm{L} 36 \mathrm{~B} 4 \mathrm{~d}, 400 \mathrm{nmol} / \mathrm{L} 36 \mathrm{~B} 4 \mathrm{u}$, and $7 \mathrm{ng}$ of genomic DNA. The thermal cycling profile was $95^{\circ} \mathrm{C}$ for $10 \mathrm{~min}$ followed by 40 cycles of $95^{\circ} \mathrm{C}$ for $15 \mathrm{sec}$ and at $56^{\circ} \mathrm{C}$ (for telomere) or $58^{\circ} \mathrm{C}$ (for $36 \mathrm{~B} 4$ ) for $1 \mathrm{~min}$. Following amplification, a dissociation curve confirmed the specificity of the reaction. During each run, negative and positive controls, a calibrator DNA sample, and a standard curve were included. For each standard curve, 2-fold serial dilutions of a reference DNA sample were used to produce a standard curve in each reaction. Two main steps were involved in telomere length quantification: first, the T/S ratio was determined for each sample based on the standard curve. Second, the ratio for each sample was normalized to the calibrator DNA to standardize sample values across all reaction plates. The laboratory personnel were blinded to disease status. $\mathrm{R}^{2}$ for each standard curve was $\geq 0.99$.

\section{Statistical Analysis}

For all subjects, the $\chi^{2}$ test was used to assess differences in the frequency distributions of the selected demographic variables and genotypes of 8 SNPs between the cases and controls. The HWE for genotype distribution in controls was tested by a goodness-of-fit $X^{2}$ test. Odds ratios (ORs) and 95\% 
confidence intervals (CIs) were calculated by univariable and multivariable unconditional logistic regression models to evaluate associations between the genotypes and risk of PCa without and with adjustment for confounding factors, respectively. Further stratification analyses were conducted to calculate the associations of SNP genotypes with $\mathrm{PCa}$ risk by demographic and clinicopathologic variables, followed by the homogeneity Q-tests. Spearman rank correlation was used to investigate associations between telomere length and age. Telomere length was categorized into dichotomies, tertile and quartile based on the distribution. To account for chance associations from multiple comparisons, we used the false-positive report probability (FPRP) to assess the false-positive association findings. All statistical analyses were performed with SAS software (version 9.1; SAS Institute, Cary, NC).

\section{Results}

\section{Characteristics of the study subjects}

The distributions of demographic characteristics of the subjects are shown in Table 1. Briefly, there were no statistical differences in the distributions of age and smoking status between two groups.

Table 1. Characteristics of PCa Cases and Controls

\begin{tabular}{llll}
\hline Variables & $\begin{array}{l}\text { Cases (\%) } \\
\mathrm{N}=1015\end{array}$ & $\begin{array}{l}\text { Controls }(\%) \\
\mathrm{N}=1052\end{array}$ & $P$ \\
\hline Age & $69.1 \pm 8.2$ & $68.6 \pm 8.9$ & 0.828 \\
$\leq 64$ & $291(28.7)$ & $308(29.3)$ & \\
$65-75$ & $496(48.9)$ & $500(47.5)$ & \\
$>75$ & $228(22.5)$ & $244(23.2)$ & \\
Smoking status & & & \\
Never & $406(40.0)$ & $412(39.2)$ & 0.697 \\
Ever & $609(60.0)$ & $640(60.8)$ & \\
PSA value (ng/ml) & & & \\
$<10$ & $180(19.4)$ & & \\
$10-20$ & $195(21.0)$ & & \\
$>20$ & $552(59.6)$ & & \\
Missing & $88(8.7)$ & & \\
Gleason score & & & \\
$\leq 7$ (3+4) & $317(31.2)$ & & \\
$\geq 7(4+3)$ & $606(59.7)$ & \\
Missing & $92(9.1)$ & \\
Stage of disease & & \\
I & $5(0.5)$ & \\
II & $434(42.8)$ & \\
III & $142(14.0)$ & \\
IV & $356(35.1)$ & \\
Missing & $78(7.7)$ & \\
\hline PSA prst & & \\
\hline
\end{tabular}

PSA: prostate-specifc antigen

\section{Associations of CYPIBI SNPs with PCa Risk}

The genotype frequencies of 8 CYP1B1 SNPs and their associations with PCa risk are summarized in Table 2. The observed genotype frequencies of 8 SNPs in controls agreed with the Hardy-Weinberg equilibrium. Furthermore, the genotype distribution of CYP1B1 rs1056836 was significantly different between the cases and controls. . In multivariate logistic regression analysis, CYP1B1 rs1056836 was associated with PCa risk. Compared with CC genotype, rs1056836 variant CG/GG genotype was associated with a decreased risk of PCa (OR: 0.80, 95\% $\mathrm{CI}=0.66-0.97)$. However, no associations between the other 7 SNPs and PCa risk were observed.

Table 2. Association of CYPIBI SNPs with PCa Risk

\begin{tabular}{|c|c|c|c|c|}
\hline SNP & $\begin{array}{l}\text { Cases, No. }(\%) \\
\mathrm{n}=1015\end{array}$ & $\begin{array}{l}\text { Controls, No. }(\%) \\
\mathrm{n}=1052\end{array}$ & OR $(95 \% \mathrm{CI})$ & $P a$ \\
\hline \multicolumn{5}{|l|}{ rs9341266 } \\
\hline GG & $774(76.3)$ & $816(77.6)$ & 1.00 & \\
\hline AG & $218(21.5)$ & $215(20.4)$ & $1.07(0.87-1.32)$ & 0.533 \\
\hline AA & $23(2.3)$ & $21(2.0)$ & $1.15(0.63-2.10)$ & 0.649 \\
\hline AG/AA vs. GG & & & $1.08(0.88-1.32)$ & 0.477 \\
\hline GG/AG vs. AA & & & $1.13(0.62-2.06)$ & 0.683 \\
\hline \multicolumn{5}{|l|}{ rs162549 } \\
\hline TT & 787 (77.5) & $811(77.1)$ & 1.00 & \\
\hline AT & $209(20.6)$ & $222(21.1)$ & $0.95(0.77-1.18)$ & 0.636 \\
\hline AA & $19(1.9)$ & $19(1.8)$ & $1.01(0.53-1.93)$ & 0.971 \\
\hline AT/AA vs. TT & & & $0.96(0.78-1.17)$ & 0.660 \\
\hline TT/AT vs. AA & & & $1.02(0.54-1.95)$ & 0.944 \\
\hline \multicolumn{5}{|l|}{ rs10916 } \\
\hline TT & 808 (79.6) & $856(81.4)$ & 1.00 & \\
\hline GT & $192(18.9)$ & $183(17.4)$ & $1.12(0.89-1.40)$ & 0.333 \\
\hline GG & $15(1.5)$ & $13(1.2)$ & $0.75(0.50-1.11)$ & 0.152 \\
\hline GT/GG vs. TT & & & $1.13(0.91-1.40)$ & 0.289 \\
\hline TT/GT vs. GG & & & $1.21(0.57-2.56)$ & 0.617 \\
\hline \multicolumn{5}{|l|}{ rs162562 } \\
\hline AA & $695(68.5)$ & $711(67.6)$ & 1.00 & \\
\hline $\mathrm{AC}$ & $287(28.3)$ & $305(29.0)$ & $0.97(0.80-1.17)$ & 0.719 \\
\hline $\mathrm{CC}$ & $33(3.3)$ & $36(3.4)$ & $0.96(0.59-1.55)$ & 0.854 \\
\hline AC/CC vs. AA & & & $0.96(0.80-1.16)$ & 0.700 \\
\hline CC/AC vs.CC & & & $0.97(0.60-1.56)$ & 0.887 \\
\hline \multicolumn{5}{|l|}{ rs2551188 } \\
\hline $\mathrm{CC}$ & $541(53.3)$ & $558(53.0)$ & 1.00 & \\
\hline $\mathrm{CT}$ & $423(41.7)$ & $421(40.0)$ & $1.05(0.88-1.26)$ & 0.564 \\
\hline TT & $51(5.0)$ & $73(6.9)$ & $0.72(0.49-1.05)$ & 0.086 \\
\hline CT/TT vs. CC & & & $1.00(0.84-1.19)$ & 0.964 \\
\hline CC/CT vs.TT & & & $0.70(0.49-1.02)$ & 0.060 \\
\hline \multicolumn{5}{|l|}{ rs9341250 } \\
\hline $\mathrm{CC}$ & 955 (94.1) & $985(93.6)$ & 1.00 & \\
\hline $\mathrm{AC}$ & $58(5.7)$ & $64(6.1)$ & $0.96(0.66-1.38)$ & 0.806 \\
\hline AA & $2(0.2)$ & $3(0.3)$ & $0.66(0.11-3.94)$ & 0.644 \\
\hline AC/AA vs. CC & & & $0.94(0.66-1.35)$ & 0.741 \\
\hline $\mathrm{CC} / \mathrm{AC}$ vs. AA & & & $0.66(0.11-3.95)$ & 0.646 \\
\hline \multicolumn{5}{|l|}{ rs1056827 } \\
\hline $\mathrm{CC}$ & $668(65.8)$ & $679(64.5)$ & 1.00 & \\
\hline $\mathrm{AC}$ & 303 (29.9) & $327(31.1)$ & $0.94(0.78-1.14)$ & 0.543 \\
\hline AA & $44(4.3)$ & $46(4.4)$ & $0.98(0.64-1.50)$ & 0.908 \\
\hline AC/AA vs. CC & & & $0.95(0.79-1.14)$ & 0.555 \\
\hline $\mathrm{CC} / \mathrm{AC}$ vs. AA & & & $0.99(0.65-1.52)$ & 0.976 \\
\hline \multicolumn{5}{|l|}{ rs1056836 } \\
\hline $\mathrm{CC}$ & $728(71.7)$ & $705(67.0)$ & 1.00 & \\
\hline CG & $274(27.0)$ & $323(30.7)$ & $0.82(0.68-0.99)$ & 0.041 \\
\hline GG & $13(1.3)$ & $24(2.3)$ & $0.54(0.27-1.07)$ & 0.079 \\
\hline CG/GG vs. CC & & & $0.80(0.66-0.97)$ & 0.020 \\
\hline CC/CG vs. GG & & & $0.57(0.29-1.14)$ & 0.111 \\
\hline
\end{tabular}

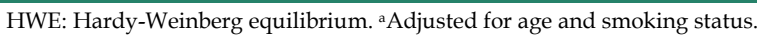

The results were in bold if $P<0.05$. 
Table 3. Association between CYPIBI SNPs and RTL

\begin{tabular}{|c|c|c|c|c|c|}
\hline SNP & Genotype & Short RTL, n (\%) & Long RTL, n (\%) & OR $(95 \% \text { CI })^{a}$ & $P$ \\
\hline \multirow[t]{2}{*}{ rs9341266 } & GG & $58(27.9)$ & $64(29.4)$ & 1.00 & \\
\hline & AG/AA & $150(72.1)$ & 154 (70.6) & $1.08(0.71-1.64)$ & 0.737 \\
\hline \multirow[t]{2}{*}{ rs162549 } & TT & $44(21.2)$ & $48(22.0)$ & 1.00 & \\
\hline & $\mathrm{AT} / \mathrm{AA}$ & $164(78.8)$ & $170(78.0)$ & $1.05(0.66-1.67)$ & 0.828 \\
\hline \multirow[t]{2}{*}{ rs10916 } & TT & $35(16.8)$ & $54(24.8)$ & 1.00 & \\
\hline & GT/GG & $173(83.2)$ & $164(75.2)$ & $1.63(1.01-2.62)$ & 0.045 \\
\hline \multirow[t]{2}{*}{ rs162562 } & AA & $60(28.8)$ & $66(30.3)$ & 1.00 & \\
\hline & $\mathrm{AC} / \mathrm{CC}$ & 148 (71.2) & $152(69.7)$ & 1.07 (0.71-1.62) & 0.747 \\
\hline \multirow[t]{2}{*}{ rs 2551188} & $\mathrm{CC}$ & $82(39.4)$ & $103(47.2)$ & 1.00 & \\
\hline & $\mathrm{CT} / \mathrm{TT}$ & $126(60.6)$ & $115(52.8)$ & $1.38(0.94-2.02)$ & 0.104 \\
\hline \multirow[t]{2}{*}{ rs9341250 } & CC & $13(6.2)$ & $17(7.8)$ & 1.00 & \\
\hline & $\mathrm{AC} / \mathrm{AA}$ & 195 (93.8) & $201(92.2)$ & $1.27(0.60-2.68)$ & 0.533 \\
\hline \multirow[t]{2}{*}{ rs1056827 } & CC & $67(32.2)$ & $69(31.7)$ & 1.00 & \\
\hline & AC/AA & $141(67.8)$ & $149(68.3)$ & 0.98 (0.65-1.47) & 0.901 \\
\hline \multirow[t]{2}{*}{ rs1056836 } & CC & $67(32.2)$ & $50(22.9)$ & & \\
\hline & CG/GG & $141(67.8)$ & 168 (77.1) & $1.60(1.04-2.45)$ & 0.033 \\
\hline
\end{tabular}

RTL: Relative telomere length adjusted for age and smoking status.

In the stratified analysis, the decreased risk was also observed in men who were ever smokers, Gleason score $\geq 7(4+3)$, and stage III/IV (Supplementary Table 1). Further homogeneity tests suggested, however, that there were no differences in the risk estimates between these strata.

\section{Genetic variations and telomere length}

To investigate whether the telomere length had prognostic significance, 426 patients were categorized into dichotomies, tertile and quartile based on their telomere length distribution (range, 0.06-2.06) (Supplementary Table 2). Telomere length was inversely associated with age $(\mathrm{r}=-0.40, P<0.001)$. When participants were dichotomized according to the median telomere length value, significant differences in telomere length by genotype of CYP1B1 rs1056836 and rs10916 were observed (Table 3). Longer telomere length showed a significantly higher proportion of the CG/GG genotypes, compared with that of the CC genotype (OR: 1.60, 95\% CI: 1.04-2.45).

\section{FPRP Values for Associations between PCa Risk and CYPIBI SNPs}

The FPRP values at different prior probability levels for all significant findings are summarized in the Supplementary Table 3. When the assumption of prior probability was 0.01 , no association was noteworthy in all subgroups for rs1056836 as well as rs10916. In contrast, some greater FPRP values for the other significant associations between CYP1B1 variants and PCa risk suggested some possible false-positive results in the findings, which need further validation in larger studies.

\section{Discussion}

In the current study we found a CYP1B1 inherited variation rs1056836 associated with PCa risk and further showed its biologic association with leukocyte telomere length. Findings on CYP1B1 polymorphisms and susceptibility to $\mathrm{PCa}$ have proved inconsistent across different ethnic groups ${ }^{[8-14]}$. However, previous studies were conducted in small-sized populations, and require confirmation in larger studies.

The SNP rs1056836 is a non-synonymous polymorphism in exon 3, which encodes the heme binding domain, and results in a G-to-C and subsequent amino acid substitutions of Valine-to-Leucine[15].The SNP has documented functional consequences on transcription, protein structure, and enzyme action ${ }^{[16,17]}$. A number of studies have reported a decreased risk of PCa for carriers of the rs1056836 G allele, but the results were inconsistent in different ethnic groups. Meta-analysis results showed that rs1056836 is significantly associated with PCa susceptibility among Asians $[18,19]$ and our findings support this association. These inconclusive results may have several causes. First, it may result from the distribution of allele frequencies among different ethnicities or between different case sources. We found that the frequency of rs1056836 G allele was significantly lower in Asian controls than in Caucasian or mixed population controls. Second, the sample sizes of the cases and controls in the Asian populations were both noticeably smaller than those in Caucasians or mixed population subjects ${ }^{[18,19]}$. The current study has sufficient power confirm the positive associations in Asians. Moreover, the mechanism of PCa development is similar to other cancers and is dependent on the interactions of genetic factors and environmental agents ${ }^{[20]}$. Environments and lifestyles are very different among individuals of different races. Thus, the aetiology of PCa is unlikely to be explained solely by genetic polymorphisms because hereditary variation alone 
cannot affect the risk of any disease. CYP1B1 rs1056836 was associated with CYP1B1 mRNA expression ${ }^{[6]}$ and overlapped with PCa risk in the expected opposite direction suggesting potential biological consequences. Furthermore, a recent study showed that overexpression of CYP1B1 stimulated proliferative, migratory and invasive potential of non-tumorigenic PCa cells ${ }^{[21]}$.

However, exact mechanisms of how CYP1B1 polymorphism contributes to $\mathrm{PCa}$ susceptibility requires further illustration. The present dataset offers an attractive possibility to address interactions between telomere length and genotypes. We hypothesized that genetic factors implicated in estrogen metabolism pathways contribute to increased oxidative stress and thereby influence telomere length. Oxidative stress causes telomere attrition by several potential pathways. Reactive oxygen species can directly damage the sensitive triple-G containing telomeres ${ }^{[22]}$, or indirectly, act by inhibiting telomerase activity and increasing the load of pro-inflammatory cytokines that accelerate immune cell turnover and hence telomere loss ${ }^{[23]}$. Since estrogen metabolism is a well-known source of reactive oxygen species formation, aberrations in this pathway could compromise the redox buffering capacity of cells ${ }^{[4]}$, which may in turn lead to increased oxidative burden and telomere attrition. Accordingly, in our study, we show for the first time that functionally relevant SNP in CYP1B1 gene impacts telomere length in PCa patients. CYP1B1 participates in the first step of estrogen metabolism, i.e., the conversion of estrogens to 2- or 4-hydroxyestrogens, and specifically catalyzes the 4-hydroxylation of estrogens. 4-hydroxyestradiol is, in sharp contrast to 2-hydroxyestradiol, inactivated by COMT at a slower rate, and undergoes metabolic redox cycling to generate free radicals and reactive semiquinone and quionone intermediates ${ }^{[24]}$. The CYP1B1 1056836 variant allele codes for the enzyme with 2-fold higher mRNA compared to wild-type allele[6], leading to increased 4-hydroxyestradiol production. Consistent with this notion, telomere length was found significantly shorter in individuals carrying the CYP1B1 rs1056836 wild type allele.

A large number of intrinsic and extrinsic factors, such as heredity, epigenetics, aging, stress, immune components, and hormones are all states have been previously associated with shorter telomere length ${ }^{[25]}$. As such, limitations of the study are mainly related to the scarcity of the relevant exposure data and nature of retrospective study design. The current study was based on the leukocytes telomere length only and not on the prostate tissue telomere length. Because there is an intra-individual synchrony in telomere length across the somatic tissues of humans as evidenced by the strong correlations between the telomere lengths in all tissue types[26]. Moreover, the rates of telomere shortening are similar in the somatic tissues. However, future work aims to directly describe the relationships between prostate tissue, leukocytes telomere lengths and PCa cases. Strengths of the study include a significant number of patients, a candidate gene approach, the high plausibility of the association based on the biologic function of selected candidate genes and an impact on telomere length associated with positive markers.

In summary, significant association with $\mathrm{PCa}$ risk for CYP1B1 rs1056836 with telomere length may provide a biological basis for the results obtained in various studies investigating the impact of CYP1B1 SNPs on PCa risk. With these implications, further investigation of SNP-cancer risk associations in specific subsets of patients with particular environmental or genetic backgrounds, as well as more detailed in vitro and in vivo biological functional studies will be helpful to elucidate how exactly the CYP1B1 genetic variations influenced $\mathrm{PCa}$ development.

\section{Abbreviations}

PSA: prostate-specific antigen; SNP: single nucleotide polymorphism; OR: odds ratio; CI: confidence interval.

\section{Supplementary Material}

Supplementary tables.

http://www.jcancer.org/v09p0269s1.pdf

\section{Competing Interests}

The authors have declared that no competing interest exists.

\section{References}

[1] Blackburn E H, Greider C W, Szostak J W. Telomeres and telomerase: the path from maize, Tetrahymena and yeast to human cancer and aging. Nat Med. 2006; 12(10):1133-1138.

[2] Benetos A, Dalgard C, Labat C, et al. Sex difference in leukocyte telomere length is ablated in opposite-sex co-twins. Int J Epidemiol. 2014; 43(6):1799-1805.

[3] Song J Y, Kim M J, Jo H H, et al. Antioxidant effect of estrogen on bovine aortic endothelial cells. J Steroid Biochem Mol Biol. 2009; 117(1-3):74-80.

[4] Yue W, Santen R J, Wang J P, et al. Genotoxic metabolites of estradiol in breast: potential mechanism of estradiol induced carcinogenesis. J Steroid Biochem Mol Biol. 2003; 86(3-5):477-486.

[5] Gajjar K, Martin-Hirsch P L, Martin F L. CYP1B1 and hormone-induced cancer. Cancer Lett. 2012; 324(1):13-30.

[6] Gu C Y, Qin X J, Qu Y Y, et al. Genetic variants of the CYP1B1 gene as predictors of biochemical recurrence after radical prostatectomy in localized prostate cancer patients. Medicine (Baltimore). 2016; 95(27):e4066.

[7] Gu C, Qu Y, Zhang G, et al. A single nucleotide polymorphism in ADIPOQ predicts biochemical recurrence after radical prostatectomy in localized prostate cancer. Oncotarget. 2015; 6(31):32205-32211.

[8] Cussenot O, Azzouzi A R, Nicolaiew N, et al. Combination of polymorphisms from genes related to estrogen metabolism and risk of prostate cancers: the hidden face of estrogens. J Clin Oncol. 2007; 25(24):3596-3602. 
[9] Beuten J, Gelfond J A, Byrne J J, et al. CYP1B1 variants are associated with prostate cancer in non-Hispanic and Hispanic Caucasians. Carcinogenesis. 2008; 29(9):1751-1757.

[10] Sobti R C, Onsory K, Al-Badran A I, et al. CYP17, SRD5A2, CYP1B1, and CYP2D6 gene polymorphisms with prostate cancer risk in North Indian population. DNA Cell Biol. 2006; 25(5):287-294.

[11] Fukatsu T, Hirokawa Y, Araki T, et al. Genetic polymorphisms of hormone-related genes and prostate cancer risk in the Japanese population. Anticancer Res. 2004; 24(4):2431-2437.

[12] Chang B L, Zheng S L, Isaacs S D, et al. Polymorphisms in the CYP1B1 gene are associated with increased risk of prostate cancer. Br J Cancer.2003; 89(8):1524-1529.

[13] Tanaka Y, Sasaki M, Kaneuchi M, et al. Polymorphisms of the CYP1B1 gene have higher risk for prostate cancer. Biochem Biophys Res Commun. 2002; 296(4):820-826.

[14] Cicek M S, Liu X, Casey G, et al. Role of androgen metabolism genes CYP1B1, PSA/KLK3, and CYP11alpha in prostate cancer risk and aggressiveness. Cancer Epidemiol Biomarkers Prev. 2005; 14(9):2173-2177.

[15] Holt S K, Kwon E M, Fu R, et al. Association of variants in estrogen-related pathway genes with prostate cancer risk. Prostate. 2013; 73(1):1-10.

[16] Bieche I, Narjoz C, Asselah T, et al. Reverse transcriptase-PCR quantification of mRNA levels from cytochrome (CYP)1, CYP2 and CYP3 families in 22 different human tissues. Pharmacogenet Genomics. 2007; 17(9):731-742.

[17] Beuten J, Gelfond J A, Franke J L, et al. Single and multigenic analysis of the association between variants in 12 steroid hormone metabolism genes and risk of prostate cancer. Cancer Epidemiol Biomarkers Prev. 2009; 18(6):1869-1880.

[18] Yang J, Xu D L, Lu Q, et al. Prostate cancer risk and aggressiveness associated with the CYP1B1 4326C/G (Leu432Val) polymorphism: a meta-analysis of 2788 cases and 2968 controls. Asian J Androl. 2012; 14(4):560-565.

[19] Zhang H, Li L, Xu Y. CYP1B1 polymorphisms and susceptibility to prostate cancer: a meta-analysis. PLoS One. 2013; 8(7):e68634.

[20] Pfeifer G P. Environmental exposures and mutational patterns of cancer genomes. Genome Medicine. 2010; 2(8):54.

[21] Chang I, Mitsui Y, Kim S K, et al. Cytochrome P450 1B1 inhibition suppresses tumorigenicity of prostate cancer via caspase-1 activation. Oncotarget. 2017; 8(24):39087-39100.

[22] Kawanishi S, Oikawa S. Mechanism of telomere shortening by oxidative stress. Ann N Y Acad Sci. 2004; 1019:278-284.

[23] Haendeler J, Hoffmann J, Brandes R P, et al. Hydrogen peroxide triggers nuclear export of telomerase reverse transcriptase via Src kinase family-dependent phosphorylation of tyrosine 707. Mol Cell Biol. 2003; 23(13):4598-4610.

[24] Tsuchiya Y, Nakajima M, Yokoi T. Cytochrome P450-mediated metabolism of estrogens and its regulation in human. Cancer Lett. 2005; 227(2):115-124.

[25] Kaszubowska L. Telomere shortening and ageing of the immune system. J Physiol Pharmacol. 2008; 59 (Suppl 9):169-186.

[26] Daniali L, Benetos A, Susser E, et al. Telomeres shorten at equivalent rates in somatic tissues of adults. Nat Commun. 2013; 4:1597. 